\title{
Establishing the utility of Recursive Partitioning Analysis for patients with intra-cranial metastases managed in a KwaZulu-Natal state sector Oncology unit
}

\begin{tabular}{|c|c|}
\hline \multicolumn{2}{|c|}{$\begin{array}{l}\text { Authors: } \\
\text { Presha Bipath }{ }^{1} \\
\text { Laura W. Stopforth }^{1} \\
\text { Santuri Naicker } \\
\text { Poovandren Govender } \\
\text { Pilbert Sibanda } \\
\text { Louise Walker }^{1} \text { (]) }\end{array}$} \\
\hline \multicolumn{2}{|c|}{$\begin{array}{l}\text { Affiliations: } \\
\text { 'Department of Oncology, } \\
\text { Faculty of Clinical Medicine, } \\
\text { University of KwaZulu-Natal, } \\
\text { Durban, South Africa }\end{array}$} \\
\hline \multicolumn{2}{|c|}{$\begin{array}{l}\text { 2Department of Biostatistics, } \\
\text { Faculty of Clinical Medicine, } \\
\text { University of KwaZulu-Natal, } \\
\text { Durban, South Africa }\end{array}$} \\
\hline \multicolumn{2}{|c|}{$\begin{array}{l}\text { Corresponding author: } \\
\text { Presha Bipath, } \\
\text { bipathp@gmail.com }\end{array}$} \\
\hline $\begin{array}{l}\text { Dates: } \\
\text { Received: } 07 \text { I } \\
\text { Accepted: } 25 \\
\text { Published: } 23\end{array}$ & $\begin{array}{l}\text { Mar. } 2021 \\
\text { une } 2021 \\
\text { Nov. } 2021\end{array}$ \\
\hline \multicolumn{2}{|c|}{$\begin{array}{l}\text { How to cite this article: } \\
\text { Bipath P, Stopforth LW, } \\
\text { Naicker S, Govender P, } \\
\text { Sibanda W, Walker L. } \\
\text { Establishing the utility of } \\
\text { Recursive Partitioning } \\
\text { Analysis for patients with } \\
\text { intra-cranial metastases } \\
\text { managed in a KwaZulu-Natal } \\
\text { state sector Oncology unit. S. } \\
\text { Afr. j. oncol. 2021;5(0), a175. } \\
\text { https://doi.org/10.4102/sajo. } \\
\text { v5i0.175 }\end{array}$} \\
\hline \multicolumn{2}{|c|}{$\begin{array}{l}\text { Copyright: } \\
\text { C 2021. The Authors. } \\
\text { Licensee: AOSIS. This work } \\
\text { is licensed under the } \\
\text { Creative Commons } \\
\text { Attribution License. }\end{array}$} \\
\hline \multicolumn{2}{|l|}{ Read online: } \\
\hline 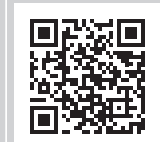 & $\begin{array}{l}\text { Scan this QR } \\
\text { code with your } \\
\text { smart phone or } \\
\text { mobile device } \\
\text { to read online. }\end{array}$ \\
\hline
\end{tabular}

Background: Brain metastases are relatively common and carry a poor prognosis. In a resource-constrained environment, the judicious use of radiotherapy must be considered in the context of its benefit. The Recursive Partitioning Analysis (RPA) scoring system is internationally validated to predict median survival in patients with brain metastases. It may be used to guide appropriate management of patients with brain metastases.

Aim: To establish the relevance of applying the RPA prognostic scoring system to the local setting.

Setting: The Department of Oncology, Greys Hospital, Pietermaritzburg, South Africa.

Methods: A retrospective chart review of patients treated for brain metastases for the period 01 January 2014 to 31 December 2019 was performed. Data was collected to determine the RPA class for each patient. Multivariate analysis of potential factors which could impact survival was done, and the actual survival of each patient was calculated.

Results: The commonest primary cancer in the study cohort was breast (67\%), followed by lung (17\%). Survival differences between RPA classes were statistically significant $(p<0.001)$. Actual survival relative to that predicted by the RPA model was 4.5 versus 7-12 months, 3.6 versus $4-7$ months, and 0.8 versus $2-4$ months, for classes I, II and III, respectively.

Conclusion: Results support the use of the RPA classification to risk stratify patients in this setting - and therefore may be used in treatment decision-making. However, it over-predicts the median survival for the local population. Larger studies are warranted in diagnostically homogenous patient groups with brain metastases, to determine survival more accurately.

Keywords: brain metastases; Recursive Partitioning Analysis; whole brain radiotherapy; prognostic scoring system.

\section{Introduction}

The most common intracranial neoplasms are metastases from an extra-cranial malignancy. ${ }^{1}$ The incidence of brain metastases is $9 \%-17 \%$ for all malignancies. Malignancies most likely to metastasise to the brain are lung $(39 \%-56 \%)$, breast $(13 \%-30 \%)$ and melanoma $(6 \%-11 \%){ }^{2}$

Brain metastases are associated with impaired quality of life and a poor prognosis: median overall survival of 2 months in untreated patients and $4-5$ months for treated patients. ${ }^{3}$

This study was conducted in an oncology unit in a tertiary public sector hospital that provides care for the hinterland of KwaZulu-Natal. A wide spectrum of malignancies is treated with breast cancer accounting for $20.4 \%$ and lung cancer $5.6 \%$ of the malignancies seen. Cervical cancer is also common accounting for $21.0 \%{ }^{4}$

Treatment modalities for brain metastases include radiotherapy, surgery and systemic therapies. ${ }^{5}$ Given the central therapeutic role that radiotherapy plays, an understanding of its appropriate use relative to actual outcomes is critical. At our centre, the main treatment modality is whole brain radiotherapy (WBRT). A range of dose fractionation regimens have been reviewed in the literature, showing similar overall survival. Fractionation regimens include 30 Gray (Gy) in 10 fractions and 20 Gy in 4 or 5 fractions. ${ }^{6}$

Prognostic scoring systems dealing with brain metastases and survival can assist clinicians in treatment decision-making. Several prognostic scoring systems have been developed to predict 
overall survival in patients with brain metastases. ${ }^{7}$ These include the Recursive Partitioning Analysis (RPA), the Basic Score for Brain Metastases (BSBM), the Rotterdam System and the Graded Prognostic Assessment (GPA). ${ }^{7}$ The various scoring systems collate individual combinations of factors into a decision-making tool to guide appropriate management. Prognostic factors include: age, Karnofsky Performance Status (KPS), presence/absence of extracranial metastases (ECM), control of the primary, number of brain metastases, and response to steroids. ${ }^{8}$ The two factors common to all scoring systems are performance status and control of extracranial disease. ${ }^{9}$

The original prognostic scoring system, the RPA, ${ }^{10}$ uses the first four factors to assign patients to three prognostic groups (class I, II or III), as shown in Table 1. Each of these classes correlates with a specific median survival range (also detailed in Table 1). The RPA index was validated in the Radiotherapy and Oncology Group database. ${ }^{11}$ The number of brain metastases was highlighted as being statistically significant on univariate analysis in the first RPA paper ${ }^{10}$, however it was omitted from the scoring system as it was not statistically significant in the final RPA analysis.

A criticism of the RPA is the exclusion of number of brain metastases from the scoring system despite the impact it has on prognosis. ${ }^{7}$ Another concern involves the inclusion of both ECM and control of the primary as prognostic factors. Their amalgamation into a single prognostic factor may be more appropriate. Other weaknesses include the extrapolation of data from three different trials (with different inclusion criteria) to devise the RPA, and the heterogeneity of patients within the three prognostic groups. ${ }^{7}$ A strength of the RPA is its applicability to a wide range of malignancies, including breast and lung cancers. ${ }^{?}$

The Rotterdam system was simplified to use three prognostic factors, but has not gained wide-spread acceptance as it includes response to steroids pre-WBRT. ${ }^{9}$ It omits control of the primary as a factor, but acknowledges ECM. ${ }^{8}$ The BSBM uses a different scoring approach and three of the four RPA criteria - omitting only age. ${ }^{7,12}$ It has been widely validated, ${ }^{8}$ but is not superior to the RPA. ${ }^{12}$ The Radiation Therapy Oncology Group (RTOG)-proposed GPA system has four prognostic tiers and uses three of the four factors used in the RPA, replacing control of primary with number of brain metastases. ${ }^{13}$ Newer prognostic systems tend to be cancer-

TABLE 1: Definition of prognostic classes of the Recursive Partitioning Analysis.

\begin{tabular}{lccc}
\hline RPA model & \multicolumn{3}{c}{ RPA class } \\
\cline { 2 - 4 } & I & II & III \\
\hline Parameter & $<65$ & $\geq 65$ & N/A \\
Age (years) & $\geq 70$ & $\geq 70$ & $<70$ \\
KPS score & Controlled & Uncontrolled & N/A \\
Control of primary & Absent & Present & N/A \\
Extracranial metastases & $7-12$ & $4-7$ & $2-4$ \\
Predicted survival (months) & &
\end{tabular}

Source: Gaspar L, Scott C, Rotman M, et al. Recursive partitioning analysis (RPA) of prognostic factors in three Radiation Therapy Oncology Group (RTOG) brain metastases trials. Int J Radiat Oncol Biol Phys. 1997;37(4):745-751. https://doi.org/10.1016/S0360-3016(96)00619-0

RPA, Recursive Partitioning Analysis; KPS, Karnofsky performance status; N/A, not applicable. type specific, such as the disease-specific GPA. ${ }^{9}$ This improves on the older prognostic systems by accommodating diseasespecific responses to systemic therapies including targeted therapy and immunotherapy, and under-representation of cancers which less commonly metastasise to the brain.

For the purposes of this study, the RPA was chosen because of ease of clinical application and known international validity $^{11}$ in a heterogenous patient group. Advanced radiotherapy techniques were not available, negating the need for a more complex prognostic system.

\section{Aim of the study}

This primary aim of the study was to establish the utility of the RPA score for patients with brain metastases being managed by a public sector oncology unit in KwaZulu-Natal. The secondary aim was to explore the correlation of individual patient and disease factors with survival.

\section{Research methods and study design}

A retrospective chart review identified patients diagnosed with brain metastases at Greys Hospital between 01 January 2014 to 31 December 2019. Inclusion criteria were histologically confirmed extracranial malignancy and radiological evidence of intracranial metastases in patients older than 12 years. Data were collected from oncology records and included age, gender, KPS at diagnosis of brain metastases, site of primary cancer, presence of extra-cranial disease, date of diagnosis of brain metastases, control of primary disease (determined clinically), and date of death. A data collection sheet was used. Each patient was assigned to an RPA class.

\section{Statistical analysis}

A multivariate analysis of factors with potential to influence prognosis was performed. Continuous variables were expressed as mean \pm standard deviation or medians (interquartile range [IQR]) and were compared using student's $t$-test. Proportions and categorical variables were compared using Pearson's chi-square test or Fisher's exact test, as appropriate. Survival estimates were calculated using KaplanMeier method, and log-rank test was used to compare survival curves. Univariate Cox regression survival analysis was used to estimate the association between survival outcome and prognostic variables. Multivariate Cox regression analysis was used to identify independent predictors of survival. All analysis was performed using Statistical Package for Social Sciences (SPSS) version 25 statistical software (IBM Corp. Released 2017. IBM SPSS Statistics for Windows, version 25.0. Armonk, NY, United States [US]).

\section{Results \\ Patient characteristics}

A total of 51 patients were included in the study, of whom 39 $(76 \%)$ were female and $12(24 \%)$ males. Most of the patients, $34(67 \%)$, had breast cancer, followed by nine (17\%) with lung 
cancer and three $(6 \%)$ with cancer of the cervix and other, five (10\%) (see Table 2). Descriptive statistics for the study population by RPA class are illustrated in Table 3. Patient ages ranged from 29 years to 78 years, with an average of 52 years and median age of 53 . Of the patients included in the study, 11 (21\%) were RPA Class III, 27 (51\%) were RPA Class II and 13 (25\%) were RPA class I. Twenty-two (22) patients $(43 \%)$ had a single brain metastasis and $42(82 \%)$ had control of their primary disease.

\section{Survival and Recursive Partitioning Analysis class}

The median survival for all the study patients who died was 3.1 months from diagnosis of brain metastases $(n=48)$ (IQR: 1.1-5.2). The median follow-up in this cohort of deceased patients was 3.1 months (IQR: 1.2-5.4). Median survival by RPA class is detailed in Table 4 . Recursive Partitioning Analysis class I patients $(n=12)$ had a median survival of 4.2 months, RPA class II patients $(n=25) 3.4$ months, and RPA class III patients $(n=11) 0.8$ months $(p<0.001)$.

Log-rank univariate and proportional hazards multivariate analyses of the data were conducted for all deceased patients in the sample (all cancers, $n=48$ ) and for those with breast cancer $(n=32)$. The results of the multivariate analysis, reported in Table 5, include the Hazard Ratios for changes in age, and KPS and RPA score. A Hazard Ratio greater than one (or a $\beta$ coefficient greater than zero) implies that a one unit increase in the factor is positively correlated with risk of death. The converse is also true. The only $\beta$ coefficient that was significantly different from zero at more than the $95 \%$ level of statistical confidence was the coefficient RPA in the analysis of all cancers. The Hazard Ratio of 2.021 for this variable indicates that an increase in RPA class is associated with a higher risk of death, that is, reduced survival. Although less statistically significant, the magnitude of the Hazard Ratios for age and KPS suggest positive relationships between these variables and survival.

\begin{tabular}{|c|c|c|c|c|c|c|}
\hline $\begin{array}{l}\text { Primary } \\
\text { site }\end{array}$ & $\begin{array}{l}\text { Number of } \\
\text { patients }\end{array}$ & $\begin{array}{c}\text { Percentage } \\
\text { of patients } \\
(\%)\end{array}$ & $\begin{array}{l}\text { Median } \\
\text { survival } \\
\text { in days }\end{array}$ & $95 \% \mathrm{Cl}$ & $\begin{array}{l}\text { Standard } \\
\text { error }\end{array}$ & $\begin{array}{c}\text { Log-rank } \\
p \text {-value }\end{array}$ \\
\hline Breast & 35 & 68.6 & 63 & $13.5-112.5$ & 25.26 & 0.117 \\
\hline Lung & 9 & 17.6 & 96 & $93.1-98.9$ & 1.49 & - \\
\hline Other & 7 & 13.7 & 105 & $37.8-172.2$ & 34.29 & - \\
\hline Overall & 51 & 100 & 93 & $51.0-135$ & 21.4 & - \\
\hline
\end{tabular}

$\mathrm{Cl}$, confidence interval.
Kaplan Meier survival curves, seen in Figure 1, show distinct separation between classes II and III as expected. Minimal separation is noted between classes I and II. As noted in Table 5, the difference in survival across the three classes of this study cohort is statistically significant $(p<0.001)$.

\section{Recursive Partitioning Analysis class, treatment and survival}

Overall, 26 patients $(n=51)(51 \%)$ received WBRT of which seven were RPA class I, 18 were RPA class II and one was RPA class III. All patients received supportive care with steroids as per local protocol. Mean survival of those patients in class I and II who died after receiving WBRT was 8.2 months for class I patients and 5.8 months for class II patients. Patients who did not receive WBRT had a mean survival of 1.9 and 3.3 months, respectively.

\section{Discussion}

The primary aim of the study was to establish the utility of the RPA classification in patients with brain metastases treated at Greys Hospital. This is the first study in South Africa to look at the use of the RPA prognostication scoring system in the local population. The model showed statistical significance at the $95 \%$ confidence level for patients with

TABLE 4: Survival by Recursive Partitioning Analysis class.

\begin{tabular}{lccc}
\hline RPA class & $\begin{array}{c}\text { Median survival } \\
\text { (months) }\end{array}$ & $\begin{array}{c}95 \% \text { confidence } \\
\text { interval }\end{array}$ & $p$ \\
\hline I $(n=12)$ & 4.2 & $0.5-7.9$ & $<0.001$ \\
II $(n=25)$ & 3.4 & $2.6-4.1$ & - \\
III $(n=11)$ & 0.8 & $0.5-1.2$ & - \\
\hline
\end{tabular}

RPA, Recursive Partitioning Analysis.

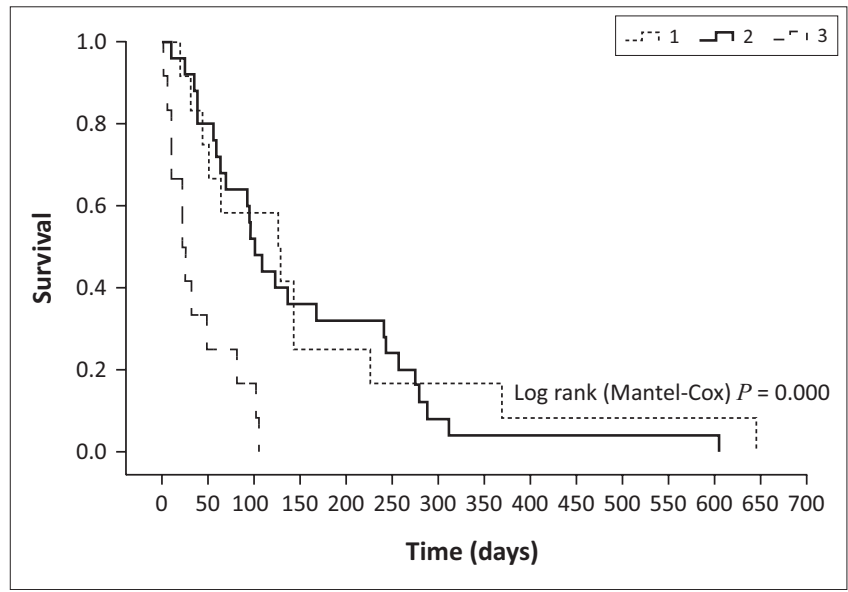

FIGURE 1: Kaplan-Meier curves of the study population survival by RTOG Recursive Partitioning Analysis score.

TABLE 3: Details of study cohort by Recursive Partitioning Analysis class.

\begin{tabular}{|c|c|c|c|c|c|c|c|c|c|c|c|c|c|c|c|c|c|c|}
\hline \multirow[t]{3}{*}{ RPA } & \multirow[t]{3}{*}{$n$} & \multirow{3}{*}{$\begin{array}{c}\text { Age } \\
\text { Mean } \pm \text { s.d. } \\
\text { (years) }\end{array}$} & \multicolumn{4}{|c|}{ KPS } & \multicolumn{4}{|c|}{ Extra-cranial disease } & \multicolumn{4}{|c|}{$\begin{array}{l}\text { Single versus multiple brain } \\
\text { metastases }\end{array}$} & \multicolumn{4}{|c|}{ Control of primary } \\
\hline & & & \multicolumn{2}{|c|}{$<70$} & \multicolumn{2}{|c|}{$\geq 70$} & \multicolumn{2}{|c|}{ Yes } & \multicolumn{2}{|c|}{ No } & \multicolumn{2}{|c|}{ Single } & \multicolumn{2}{|c|}{ Multiple } & \multicolumn{2}{|c|}{ Yes } & \multicolumn{2}{|c|}{ No } \\
\hline & & & $n$ & $\%$ & $n$ & $\%$ & $n$ & $\%$ & $n$ & $\%$ & $n$ & $\%$ & $n$ & $\%$ & $n$ & $\%$ & $n$ & $\%$ \\
\hline 1 & 13 & $51 \pm 12$ & 0 & 0 & 13 & 100 & 0 & 0 & 13 & 100 & 8 & 62 & 5 & 38 & 13 & 100 & 0 & 0 \\
\hline II & 27 & $51 \pm 14$ & 0 & 0 & 27 & 100 & 21 & 78 & 6 & 22 & 11 & 42 & 15 & 58 & 19 & 70 & 8 & 30 \\
\hline III & 11 & $55 \pm 16$ & 12 & 100 & 0 & 0 & 3 & 25 & 9 & 75 & 3 & 27 & 8 & 73 & 10 & 83 & 2 & 17 \\
\hline
\end{tabular}

RPA, Recursive Partitioning Analysis; s.d., standard deviation; KPS, Karnofsky Performance Status. 
TABLE 5: Univariate proportional hazards of continuous variables affecting survival in patients with brain metastases.

\begin{tabular}{llcccc}
\hline Cancer & Variable & Coefficient $(\boldsymbol{\beta})$ & $\begin{array}{c}\text { Standard } \\
\text { error }\end{array}$ & Hazard ratio & $\begin{array}{c}\text { Model } \\
\text { significance }(\boldsymbol{p})\end{array}$ \\
\hline Breast & Age & -0.013 & 0.017 & 0.989 & 0.02 \\
& KPS & $-0.051^{*}$ & 0.026 & 0.951 & - \\
& RPA Score & $0.417^{* *}$ & 0.388 & 1.518 & - \\
All cancers & Age & -0.013 & 0.011 & 0.987 & 0.07 \\
& KPS & -0.019 & 0.012 & 2.260 & - \\
& RPA Score & $0.704^{* * *}$ & 0.271 & 2.021 & - \\
\hline
\end{tabular}

RPA, Recursive Partitioning Analysis; KPS, Karnofsky Performance Status.

$*, * *$ and $* * *$ denote statistical significance at the $90 \%, 95 \%$ and $99 \%$ levels of statistical confidence, respectively.

breast cancer $(p=0.02)$ and $90 \%$ confidence level when applied to all diagnoses $(p=0.07)$ (see Table 5). That said, the survival of patients in the different RPA classes in our study was markedly different from that predicted by the model ${ }^{10}$ (see Table 4), and this needs to be recognised when being used in clinical practice. Possible reasons for this discrepancy are detailed below.

The study population differs socio-demographically from that in the RTOG trials (principally conducted in the developed world). The majority of patients in the areas served by the oncology centre are rural, have lower education levels and are of a lower socio-economic status. ${ }^{14}$ The intensity of poverty in the area is $42.6 \%{ }^{15}$ Cačala and Gilart ${ }^{16}$ in the same clinical setting found that breast cancer patients with lower levels of formal education presented with a more advanced tumour $(p<0.05)$. The average level of education was grade six with $19 \%$ of their patient cohort having no formal education. Furthermore, $77.0 \%$ of study patients were unemployed. Makhatini et al. ${ }^{17}$ while reviewing patients with gestational trophoblastic neoplasms from the same clinical setting found that rural patients living further from the oncology centre had statistically significant levels of poor compliance with treatment and correspondingly worse outcomes. ${ }^{17}$

A study of patients with brain metastases in the United States reviewed potential non-clinical determinants of cancer outcomes. ${ }^{18}$ It showed that patients with a lower estimated income were likely to experience a higher symptom burden as a result of their metastases in the present as well as later. In this population, however, all-cause mortality and death because of brain metastases were not statistically significantly impacted by rural-urban location or access to health insurance. As discussed above, this is in contrast with South African studies from KwaZulu-Natal. ${ }^{16,17}$ Limited resources and a lack of oncology outreach at peripheral institutions may also delay diagnosis of either primary or metastatic/ recurrent disease and contribute to poorer survival rates.

Recursive Partitioning Analysis class III had the worst prognosis - median survival 0.8 months (Table 4). Whilst for RPA class III patients, having the poorest prognosis in keeping with the RPA model, the median survival in our study cohort was worse than predicted. This is similar to the overall survival discrepancy, and the perception is that the contributory factors are similar. A review of Table 4 shows that RPA Class II and I patients fared better than those in class III. This is again consistent with the findings of the original RPA papers. As expected from the RPA database, those in class II had an intermediate prognosis which was worse than patients classified as class I. The separation of survival curves for RPA classes II and III is clear in Figure 1. Survival curves for RPA classes I and II do not separate, contrary to a priori expectations. This may be attributed to the small sample size. Again, while relative prognosis of RPA class I and II patients is consistent with the original RPA cohort, the actual survival of even RPA class I and II patients was worse than predicted. Apart from the factors described above, the poorer survival of RPA class I and II patients in this study could be, at least in part, as a result of lower uptake of radiotherapy (only $68 \%$ of class I \& II patients received radiotherapy). Radiotherapy uptake will be discussed in greater detail below. In summary, while RPA classes adequately stratify prognostic predictions, these predictions seem to be overly optimistic for our setting.

Recursive Partitioning Analysis studies suggest worse prognosis for patients with poorer KPS and older age. ${ }^{10,11}$ In this study, the individual contributions of KPS and age to overall survival was not statistically significant (see Table 5), which may imply that socio-economic factors contributed to a greater extent than in the model. This would be an interesting topic for further research. It is equally possible that the lack of statistical significance may be because of the small sample size.

Disease profiles of study patients (see Table 2) differ from that in the international RPA literature. The RPA database and the RTOG 91-04 trial reviewed a majority of lung cancer patients (66\% and $61 \%$, respectively) with $12 \%$ and $11 \%$ breast cancer patients and $21 \%$ and $23 \%$ with cancers of other sites. ${ }^{10,11}$ These had an over-representation of lung cancers (which was a criticism of the RPA studies) and an appropriate proportion of breast cancers. In this study, $18 \%$ of patients had lung cancer, $69 \%$ had breast cancer and other cancer sites were $14 \%$. This is unsurprising given the low numbers of lung cancer patients seen in our centre coupled with the high prevalence of breast cancer. ${ }^{4}$ Furthermore, the breast cancer records are wellmaintained because of ongoing disease-specific research at the centre and are therefore more accessible.

A surprising finding of our study was the unexpectedly high proportion of cervical cancer patients at $6 \%$ of the study cohort. Cancer of the Cervix does not commonly metastasise to the brain, with an incidence of $0.4 \%-2.3 \% .{ }^{19}$ While our small sample size complicates the interpretation of results, it is likely a reflection of the high burden of cervical cancer in the patient population that this unit serves. ${ }^{4}$

This study did not account for treatment and its potential influence on survival. The study cohort was unfortunately too small to allow for useful analysis of subgroups that did and did not receive radiotherapy. It is reasonable that RPA class III patients can be offered optimal supportive care 
(OSC) with steroids only in the context of a poor prognosis. In our cohort, only one RPA class III patient received radiotherapy. This is probably because of the relevance placed by doctors on KPS when making treatment decisions. Lower KPS score is a key criterion for allocation to RPA class III and may have acted as a surrogate marker. The relatively low rates of radiotherapy in RPA class I and II patients in this study (58\% and $72 \%$, respectively) is of interest and, as mentioned previously, may have contributed to the poorer than expected outcomes. Low radiotherapy uptake may be for reasons of patient reluctance, resource limitations and patients defaulting radiotherapy appointments. The sample size was too small to analyse in further detail.

Literature as far back as 1954 suggests that treatment with radiotherapy improves survival. ${ }^{20}$ However, there is a dearth of literature which confirms the benefit of WBRT over best supportive care. What is known is that reasonable fractionation schedules include $30 \mathrm{~Gy}$ in $3 \mathrm{~Gy}$ fractions and 20 Gy in 4 Gy fractions. ${ }^{6}$ No other treatment regimen has proved superior. Generally, in our practice, we tend to prescribe the longer course of palliative radiotherapy and reserve the 20 Gy regimen for patients with a worse performance status. The RTOG 91-04 triall included only RPA class I and II candidates, and all participants received radiotherapy. Different radiotherapy fractionation schedules were used. Similarity of outcomes despite the various treatment arms implied that overall survival for class I and II patients was independent of radiotherapy fractionation received.

There are only two randomised controlled trials assessing the addition of WBRT to OSC. The first, conducted in 1971, randomised 48 patients to receive prednisone alone or in combination with radiotherapy (total dose of 40 Gy in 2 Gy fractions). ${ }^{21}$ There was a marginal numerical advantage in overall survival in favour of the combination treatment arm (14 weeks vs. 10 weeks). However, no $p$-value was given, so the outcome was statistically inconclusive. The second (QUARTZ trial) ${ }^{22}$ trial was a much larger study. It aimed to show non-inferiority of OSC, including dexamethasone, to OSC and radiotherapy (20 Gy in five daily fractions) in patients with non-small cell lung primaries. The similar OS for both arms led to the conclusion that radiotherapy does not provide meaningful additional benefit to these patients. This trial has been criticised based on short median OS (8-9 weeks). It may be useful to reproduce a trial similar to the QUARTZ trial, in a fitter patient cohort, to understand better, the value of radiotherapy for patients with brain metastases regarding quality of life and OS. It is notable that the median survival for our study population was 3.1 months (13 weeks), marginally better than that for the QUARTZ study population, but still shorter than that predicted by the RTOG trials. Ultimately, the literature regarding the benefit of radiotherapy for patients with brain metastases highlights the need for careful patient selection prior to treatment - a task we feel is aided by prognostic scoring systems like the RPA.
The treatment of choice for multiple brain metastases has traditionally been WBRT. ${ }^{6}$ As treatment modalities have evolved, subgroups of patients have been identified who may benefit from surgery or stereotactic radiosurgery, with or without WBRT. ${ }^{23}$ Radiotherapy techniques and supportive therapy have also evolved such that hippocampal-sparing for WBRT is advocated. ${ }^{24}$ Memantine may be added, if available. ${ }^{25}$ Memantine is however, not available in our setting.

\section{Study strengths and limitations}

A major strength of this study is that it focuses on the local population, which is different physiologically and socioeconomically from the cohort on whom the RPA prognostication classification was based. Patients reported on in both the QUARTZ study and this study represent realworld radiotherapy practice, compared with those enrolled in the RTOG studies, where exclusion criteria may have selected for patients with potentially better treatment outcomes.

Ascertaining which patients had brain metastases during the period specified was challenging and those diagnosed outside the unit may not have been included in the study. This may have contributed to incomplete inclusion of patients. Consequently, the number of patients included in the study was smaller than expected. For this reason, the statistical analysis was unable to tease out individual variables that may impact on median OS with any degree of certainty. These factors include all the variables that underpin the RPA scoring system.

A limitation of all the RPA literature, this study included, is the heterogeneity of patients' diagnoses studied. Future RPA research, and indeed research on brain metastases in general, is likely to benefit from a disease-specific approach. This is especially true for settings where targeted agents are accessible, but applies to the resource-constrained setting as well.

\section{Practice implications}

The poor survival of all three RPA classes in this study cohort should lead doctors in similar settings to be circumspect when prescribing WBRT. Those patients considered to have a more favourable prognosis and potentially good response to radiotherapy - for example, a class I patient and select class II patients - should be considered for optimal palliative radiotherapy, for example, $30 \mathrm{~Gy}$ in 2 weeks. Patients requiring relief from neurological symptoms may be considered for alternative, shorter fractionation schedules as local resources allow. Patients with a projected poor survival would probably be best served by receiving steroids and OSC. Care should be taken to treat the patient based on their individual merit and not to resort to radiotherapy treatments purely because they are available. 


\section{Conclusion}

During the study period of 6 years, 51 patients were treated for brain metastases at Greys hospital. The majority of patients treated had a breast primary with the second largest group having a lung primary.

In our study, although RPA class did predict for survival, the survival seen in each group was less than that predicted by the RPA model. RPA class was the sole statistically significant determinant of survival, however hazard ratios suggest that age and KPS are also correlated.

Overall, this study supports the use of RPA classification to risk stratify patients in a low-middle income setting and may assist in treatment decision-making. Poorer survival in each class needs to be considered when making such decisions.

\section{Acknowledgements}

The authors would like to thank Mohammed Bapeekee for his assistance with the data collection.

\section{Competing interests}

The authors declare that they have no financial or personal relationships that may have inappropriately influenced them in writing this article.

\section{Authors' contributions}

P.B., L.W.S., S.N., P.G., and L.W. contributed to the design and implementation of the research, to the analysis of the results and to the writing of the manuscript. W.S. contribution toward the articles the statistical analyses and input.

\section{Ethical considerations}

Ethical approval was obtained from the Biomedical Research Ethics Committee (BREC) of the University of KwaZulu-Natal (BREC Number BE017/19).

\section{Funding information}

The authors received no financial support for the research, authorship, and/or publication of this article.

\section{Data availability}

The data that support the findings of this study are available from the corresponding author, P.B., upon reasonable request.

\section{Disclaimer}

The views and opinions expressed in this article are those of the authors and do not necessarily reflect the official policy or position of any affiliated agency of the authors.

\section{References}

1. Halperin EE, Perez CA, Bracy LW. Principles and practice of radiation oncology Philadelphia: Lippincot; 2019, p. 7402.

2. Schroeder $\mathrm{T}$, Bittrich $\mathrm{P}$, Kuhne JF, et al. Mapping distribution of brain metastases: Does the primary tumor matter? J Neuro-Oncol. 2020;147(1):229-235. https:// doi.org/10.1007/s11060-020-03419-6

3. Sundström JT, Minn H, Lertola KK, Nordman E. Prognosis of patients treated for intracranial metastases with whole-brain irradiation. Ann Med. 1998:30(3):296-299. https://doi.org/10.3109/07853899809005858

4. Mbeje NP, Ginindza TG, Jafta N. Establishing and evaluating cancer surveilance system in Kwa-Zulu Natal, South Africa. Cancer Infromation. 2021;20. https://doi. org/10.1177/11769351211029967

5. Tsao MN, Sultanem K, Chiu D, et al. Supportive care management of brain metastases: What is known and what we need to know. Conference Proceedings of the National Cancer Institute of Canada (NCIC) Workshop on Symptom Control in Radiation Oncology. Clin Oncol (R Coll Radiol). 2003;15(7):429-434. https://doi. in Radiation Oncology. Clin Oncol (R Coll
org/10.1016/S0936-6555(03)00220-6

6. Tsao $\mathrm{MN}$, Xu W, Wong RK, et al. Whole brain radiotherapy for the treatment of newly diagnosed multiple brain metastases. Cochrane Database Syst Rev. 2018;1(1):Cd003869. https://doi.org/10.1002/14651858.CD003869.pub4

7. Venur VA, Ahluwalia MS. Prognostic scores for brain metastasis patients: Use in clinical practice and trial design. Chin Clin Oncol. 2015;4(2):18.

8. Nieder C, Mehta MP. Prognostic indices for brain metastases--usefulness and challenges. Radiat Oncol. 2009;4:10. https://doi.org/10.1186/1748-717X-4-10

9. Viani GA, Bernardes da Silva LG, Stefano EJ. Prognostic indexes for brain metastases: Which is the most powerful? Int J Radiat Oncol Biol Phys. 2012;83(3):e325-e330. https://doi.org/10.1016/j.ijrobp.2011.12.082

10. Gaspar L, Scott C, Rotman M, et al. Recursive partitioning analysis (RPA) of prognostic factors in three Radiation Therapy Oncology Group (RTOG) brain metastases trials. Int J Radiat Oncol Biol Phys. 1997;37(4):745-751. https://doi. org/10.1016/S0360-3016(96)00619-0

11. Gaspar LE, Scott C, Murray K, Curran W. Validation of the RTOG recursive partitioning analysis (RPA) classification for brain metastases. Int J Radiat Oncol Biol Phys. 2000;47(4):1001-1006. https://doi.org/10.1016/S0360-3016(00)00547-2

12. Zindler JD, Rodrigues $\mathrm{G}$, Haasbeek $\mathrm{CJ}$, et al. The clinical utility of prognostic scoring systems in patients with brain metastases treated with radiosurgery. Radiothe Oncol. 2013;106(3):370-374. https://doi.org/10.1016/j.radonc.2013.01.015

13. Sperduto PW, Berkey B, Gaspar LE, Mehta M, Curran W. A new prognostic index and comparison to three other indices for patients with brain metastases: An analysis of 1,960 patients in the RTOG database. Int J Radiat Oncol Biol Phys. 2008;70(2):510-514. https://doi.org/10.1016/j.ijrobp.2007.06.074

14. Molongoana C, Du Plessis I, Nyeande A. Statistical release (Revised). Census 2011. Pretoria: StatsSA; 2012

15. KwaZulu-Natal Department of Health. Province of KwaZulu-Natal strategic plan 2021-2024. Pietermaritzburg: KwaZulu-Natal Department of Health.

16. Čačala SR, Gilart J. Factors relating to late presentation of patients with breast cancer in area 2 KwaZulu-Natal, South Africa. J Glob Oncol. 2017;3(5):497-501. https://doi.org/10.1200/JGO.2016.008060

17. Makhathini BS, Dreyer G, Buchmann EJ. Gestational trophoblastic disease managed at Grey's Tertiary Hospital: A five-year descriptive study. S Afr J Gynaecol Oncol. 2019;11(2):15-19. https://doi.org/10.1080/20742835.2019.1667627

18. Alphonse-Sullivan N, Taksler GB, Lycan T, et al. Sociodemographic predictors of patients with brain metastases treated with stereotactic radiosurgery. Oncotarget. 2017;8(60):101005-101011. https://doi.org/10.18632/oncotarget.22291

19. Divine LM, Kizer NT, Hagemann AR, et al. Clinicopathologic characteristics and survival of patients with gynecologic malignancies metastatic to the brain Gynecol Oncol. 2016;142(1):76-82. https://doi.org/10.1016/j.ygyno.2016.04.030

20. Chao JH, Phillips R, Nickson JJ. Roentgen-ray therapy of cerebral metastases. Cancer 1954;7(4):682-689. https://doi.org/10.1002/1097-0142(195407)7:4\%3C682::AIDCNCR2820070409\%3E3.0.CO;2-S

21. Borgelt B, Gelber R, Kramer S, et al. The palliation of brain metastases: Final results of the first two studies by the Radiation Therapy Oncology Group. Int J Radia Oncol Biol Phys. 1980;6(1):1-9. https://doi.org/10.1016/0360-3016(80)90195-9

22. Mulvenna $P$, Nankivell $M$, Barton R, et al. Dexamethasone and supportive care with or without whole brain radiotherapy in treating patients with non-smal cell lung cancer with brain metastases unsuitable for resection or stereotactic radiotherapy (QUARTZ): Results from a phase 3, non-inferiority, randomised trial. Lancet. 2016;388(10055):2004-2014. https://doi.org/10.1016/S01406736(16)30825-X

23. Cohen-Inbar O, Sheehan JP. The role of stereotactic radiosurgery and whole brain radiation therapy as primary treatment in the treatment of patients with brain oligometastases - A systematic review. J Radiosurg SBRT. 2016;4(2):79-88.

24. Oskan F, Ganswindt U, Schwarz SB, Manapov F, Belka C, Niyazi M. Hippocampus sparing in whole-brain radiotherapy. A review. Strahlenther Onkol. 2014;190(4):337-341. https://doi.org/10.1007/s00066-013-0518-8

25. Brown PD, Pugh S, Laack NN, et al. Memantine for the prevention of cognitive dysfunction in patients receiving whole-brain radiotherapy: A randomized double-blind, placebo-controlled trial. Neuro-Oncology. 2013;15(10):1429-1437 https://doi.org/10.1093/neuonc/not114 